\title{
Flebótomos (Diptera, Phlebotominae) da Ilha de São Luis, zona do Golfão Maranhense, Brasil
}

\author{
Phlebotomines (Diptera,Phlebotominae) in the Island of São Luis, \\ Big Golf zone, Maranhão State, Brazil \\ José Manuel Macário Rebêlo, Jack Any Carvalho de Araújo, Muzenilha Lira \\ Carvalho, Vera Lúcia Lopes Barros, Francinaldo Soares Silva e \\ Sueli Trindade de Oliveira
}

\begin{abstract}
Resumo Apresenta-se uma lista de flebotomíneos com 32 espécies, sendo 1 Brumptomyia e 31 Lutzomyia, distribuídas nos subgêneros Psychodopygus (6), Nyssomyia (5), Pressatia (3), Evandromyia (2), Psathyromyia (2), Sciopemyia (2), Lutzomyia (1), Micropygomyia (1), Viannamyia (1), e nos grupos Oswaldoi (5) e Migonei (3). Os flebotomíneos foram capturados na mata, peridomicílio (chiqueiro, galinheiro e estábulo) e intradomicílio (dormitório), das 18 às 6 horas, uma vez por mês, durante 4 anos na llha de São Luis, Maranhão. Todas as espécies ocorreram na mata, sendo que 16 também foram encontradas no peri e 11 dentro dos domicílios. Foram capturados 22.581 espécimens, sendo que 65,1\% ocorreram no peridomicílio, $17,5 \%$ na mata e $17,4 \%$ no intradomicílio. A espécie mais comum foi Lutzomyia longipalpis com 66,4\% dos exemplares capturados, seguida por L. whitmani (24\%) e L. evandroi $(5,9 \%)$. As 29 espécies restantes representaram apenas 3,7\% da amostra total.
\end{abstract}

Palavras-chaves: Lutzomyia. Phlebotominae. Leishmanioses.

Abstract This study lists 32 species of sand flies, 1 of them belonging to the genus Brumptomyia and 31 to the genus Lutzomyia, distributed among the following subgenera: Psychodopygus (6), Nyssomyia (5), Pressatia (3), Evandromyia (2), Psathyromyia (2), Sciopemyia (2), Lutzomyia (1), Micropygomyia (1), Viannamyia (1), and the groups Oswaldoi (5) and Migonei (3). The sand flies were captured in the wild (forest) and in peridomicile (pigpen, hen house and stable) and intradomicile (bedroom) areas from 06:00 PM to 06:00 AM, once a month, for 4 years on the Island of São Luis, Maranhão. All species sampled were present in the forest. Among them, 16 were found in the peridomicile, while 11 were found inside the houses. A total of 22,581 specimens were captured, $65.1 \%$ of them in the peridomicile, $17.5 \%$ in the forest and $17.4 \%$ in the intradomicile. The most common species was Lutzomyia longipalpis (66.4\% of the captured specimens), followed by Lutzomyia whitmani (24\%) and Lutzomyia evandroi (5.9\%). The remaining 29 species represented $3.7 \%$ of the total sample.

Key-words: Lutzomyia Phlebotominae Leishmaniasis.

Departamento de Patologia, Núcleo de Patologia Tropical e Medicina Social da Universidade Federal do Maranhão, São Luis, MA. Endereço para correspondência: Dr. José Manuel Macário Rebêlo. Deptoำ de Patologia/NPTMS/UFMA. Pça Madre Deus 2, 65025-560 São Luis, MA. Fax: 5598 232-3837.

Recebido para publicação em 5/1/98. 
A Ilha de São Luis tem sido nos últimos anos área piloto para a realização de vários projetos vinculados ao programa de controle das leishmanioses, devido ao caráter endêmico da leishmaniose visceral (LV) e ao recente surgimento de casos autóctones da leishmaniose tegumentar americana (LTA), especialmente nas periferias urbanas, áreas de ocupação da população de baixa renda, as chamadas invasões. $\mathrm{O}$ aparecimento de focos sazonais de casos de infecção, em pontos isolados da llha associa-se freqüentemente ao desmatamento desordenado da cobertura vegetal para estabelecimento de grandes projetos e aglomerados habitacionais, fato muito comum nos últimos anos ${ }^{14}$.

Para se ter uma noção da gravidade do problema, basta verificar que no período de janeiro de 1995 a abril de 1996 foram atendidos no Ambulatório do Departamento de Patologia da Universidade Federal do Maranhão, 46 pacientes portadores de LTA autóctones da llha de São Luis ${ }^{14}$. A maior prevalência foi em áreas submetidas ao impacto do desmatamento e onde, provavelmente ocorreu a peridomiciliação do vetor. Aliás, quase nada se sabe sobre a dinâmica vetorial da LTA no Maranhão. Os estudos são ainda incipientes2 312 .

A LV no Maranhão envolve um grande contingente populacional da periferia das áreas urbanas, especialmente da llha de São Luis. Fato inusitado, quando comparado ao contigente de LV das década de 50 e 60 no Brasil14. A situação epidêmica desta enfermidade no Maranhão foi instalada desde 1982, através de um surto que se estendeu até 1986, com o registro de aproximadamente 1.124 casos7. Os cães eram incriminados como reservatórios e Lutzomyia longipalpis como vetor. O grande estímulo para que este fato ocorresse, talvez tenha sido o deslocamento de grupos populacionais vindo de outras regiões do Nordeste em busca de emprego nas grandes cidades do Maranhão, principalmente sua capital São Luis, que neste período passava por uma transformação industrial com implantação de grandes projetos como a indústria de Alumínio (ALUMAR) e o corredor de exportação de Carajás da Companhia Vale do Rio Doce (CVRD)6. Naquele período, o desmatamento no interior da llha foi intenso e desordenado e implantaram-se, também, conjuntos habitacionais e outros projetos.

Em virtude das peculiaridades que envolvem a leishmaniose, as diversas formas com que ela se apresenta e o pouco conhecimento que temos acerca dos vetores e sua dinâmica, um estudo entomológico aprofundado faz-se necessário nas diversas áreas de ocorrência da doença. Assim, poder-se-ia obter uma definição precisa da fauna vetorial, indispensável para os estudos epidemiológicos e campanhas de prevenção.

Neste trabalho, apresenta-se uma lista das espécies de flebótomos que ocorrem na llha de São Luis e sua associação com as habitações humanas, seus anexos e com o ambiente silvestre.

\section{MATERIAL E MÉTODOS}

O estudo foi realizado na llha de São Luis que possui uma área territorial de $905 \mathrm{~km}^{2}$, situada no litoral setentrional brasileiro a $2^{\circ} 32^{\prime} S$

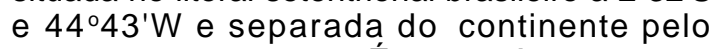
Estreito dos Mosquitos. É constituída por terras baixas (exceto a leste), elevando-se a $32 \mathrm{~m}$ de altitude onde está localizada São Luis, a capital do Estado do Maranhão. Limita-se ao norte com o oceano atlântico, a oeste com a baía de São Marcos, a leste com as baías de São José de Ribamar e do Arraial e ao sul com o Estreito dos Mosquitos 8 .

Até algum tempo atrás, a vegetação era um misto da floresta latifoliada e babaçual (Orbygnia phalerata), além da floresta paludosa marítima (os manguezais) 11. Ultimamente, a ecologia da Ilha vem-se modificando em função da demanda imobiliária e derrubadas constantes e desenfreadas das matas. Os remanescentes da vegetação original são representados por coberturas florísticas secundárias e áreas de matas baixas com intrusões de babaçu, com manchas de cerrados e formações pioneiras (campo e restinga) e, margeando os rios, onde ainda há matas ciliares, predominam os buritizais (Mauritia vinifera) e juçarais (Euterpe oleraceae).

O clima é tropical mesotérmico e úmido, com duas estações bem definidas. A chuvosa, de janeiro a junho, concentra em média, cerca de $94 \%$ do total anual das chuvas; a estação seca, de julho a dezembro, concentra apenas $6 \%$. 0 total pluviométrico é elevado em torno de $1.900 \mathrm{~mm}$ anuais, em média. As temperaturas são elevadas durante o ano todo (média de $26^{\circ} \mathrm{C}$ ) com variação anual pequena 8 .

Os flebótomos foram capturados durante 4 anos (1994-1997), das 18 às 6 horas, uma vez 
por mês, com uso de armadilhas luminosas do tipo CDC, em três áreas da Ilha. Em cada área foram estudados os ambientes de mata, peri e intradomicílio. Em cada ambiente, foi instalada uma armadilha a $1,5 \mathrm{~m}$ de altura, sempre no mesmo local. Na mata, a armadilha distava mais de $500 \mathrm{~m}$ das habitações; no peridomicílio era colocada em um anexo (chiqueiro, galinheiro ou estábulo, distantes no máximo $30 \mathrm{~m}$ da casa) e na habitação era instalada no interior de um dormitório.

Ocasionalmente utilizava-se os moradores como iscas dentro de casa e animais domésticos no peri e na mata. Nestes casos, os flebótomos eram capturados com tubo de sucção tipo Castro.

Todo material coletado foi identificado no Laboratório de Entomologia da Universidade Federal do Maranhão, pelos membros da equipe deste trabalho, auxiliados pela chave de identificação proposta por Young e Duncan 15 .

Utilizou-se teste estatístico não-paramétrico (qui-quadrado) para analisar as diferenças entre os flebótomos capturados nos diversos ambientes. As diferenças foram consideradas significativas quando a probabilidade $(p)$ do erro foi inferior a $5 \%(p<0,05)$.

\section{RESULTADOS}

Foram encontradas 1 espécie do gênero Brumptomyia França e Parrot, 1921 e 31 espécies do gênero Lutzomyia França, 1924 nos diversos ambientes da llha de São Luis distribuídas nos seguintes subgêneros: Psychodopygus (6), Nyssomyia (5), Pressatia (3), Evandromyia (2),
Psathyromyia (2), Sciopemyia (2), Lutzomyia (1), Micropygomyia (1) e Viannamyia (1). As demais espécies estão distribuídas nos grupos Oswaldoi (5) e Migonei (3). As 32 espécies estão relacionadas na lista das espécies de Phebotominae da Ilha de São Luis, MA, abaixo:
- Subgênero Brumptomyia França e Parrot, 1921

Brumptomyia avellari (Costa Lima, 1932)

- Subgênero Nyssomyia Barretto, 1962

Lutzomyia antunesi (Coutinho, 1939)

Lutzomyia flaviscutellata (Mangabeira, 1942)

Lutzomyia olmeca nociva Young e Arias, 1982

Lutzomyia umbratilis Ward e Frahia, 1977

Lutzomyia whitmani (Antunes e Coutinho, 1939)

- Subgênero Pressatia Mangabeira, 1942

Lutzomyia choti (Floch e Abonnenc, 1941)

Lutzomyia triacantha (Mangabeira, 1942)

Lutzomyia trispinosa (Mangabeira, 1942)

- Subgênero Psychodopygus Mangabeira, 1941

Lutzomyia amazonensis (Root, 1934)

Lutzomyiaclaustrei Abonnenc, Léger e Fauran, 1979

Lutzomyia corossoniensis Le Pont e Pajot, 1978

Lutzomyia davisi (Root, 1934)

Lutzomyia hirsutus (Mangabeira, 1942)

Lutzomyia wellcomei Fraiha, Shaw e Lainson, 1971

- Subgênero Evandromyia Mangabeira, 1941

Lutzomyia bourrouli (Barretto e Coutinho, 1941)

Lutzomyia infraspinosa (Mangabeira, 1941)

Na Tabela 1, estão relacionadas as espécies e os números de espécimens capturados em cada ambiente. Das 32 espécies encontradas
- $\quad$ Subgênero Lutzomyia França, 1924

Lutzomyia longipalpis (Lutz e Neiva, 1912)

- Subgênero Micropygomyia Barretto, 1962

Lutzomyia micropyga (Mangabeira, 1942)

- Subgênero Psathyromyia Barretto, 1962

Lutzomyia shannoni (Dyar, 1929)

Lutzomyia dendrophyla (Mangabeira, 1942)

- Subgênero Sciopemyia Barretto, 1962

Lutzomyia sordellii (Shannon e Del Ponte, 1927)

Lutzomyia fluviatilis (Floch e Abonnenc, 1944)

- Subgênero Viannamyia Mangabeira, 1941

Lutzomyia furcata (Mangabeira, 1941)

- Grupo Migonei

Lutzomyia evandroi (Costa Lima e Antunes, 1936)

Lutzomyia migonei (França, 1920)

- Grupo Oswaldoi

Lutzomyia termitophila Martins, Falcão e Silva, 1964

Lutzomyia oswaldoi (Mangabeira, 1942)

Lutzomyia trinidadensis (Newstead, 1922)

Lutzomyia longipennis (Barretto, 1946)

Lutzomyia rorotaensis (Floch e Abonnenc, 1944)

na Ilha, todas estiveram presentes na mata, sendo que 16 restringiram sua ocorrência a este ambiente, enquanto as outras $16(50 \%)$ foram 
encontradas também no peri. Destas, 11 (34\%) foram capturadas dentro das habitações humanas (Tabela 1).

Em relação ao número de espécimens, foram capturados 22.581 exemplares, sendo $65,1 \%$ no peridomicílio, $17,5 \%$ na mata e $17,4 \%$ no intradomicílio (Figura 1).

Entre as espécies encontradas, exclusivamente na mata, as mais abundantes foram $L$. infraspinosa, $L$. sordellii e $B$. avellari. Já entre aquelas que se distribuíram nos três ambientes destacaram-se L. longipalpis, L. whitmani, L. evandroi e L. flaviscutellata.

L. longipalpis foi a espécie mais comum, representando $66,4 \%$ dos flebótomos capturados ou seja 14.987 exemplares (Figura 2), sendo mais freqüente no ambiente domiciliar, peri
(78\%) e intra (16,6\%) (Figura 3). Entretanto, o número de espécimens coletados no peri foi significativamente maior do que no intra $(p<0,05)$.

L. whitmani foi a segunda espécie mais abundante com $24 \%$ dos espécimens (5.440 exemplares), ocorrendo em alta freqüência no peridomicílio $(42,5 \%)$ e na mata $(44,6 \%)$, não havendo diferença significativa entre estes ambientes $(p<0,05)$. Entretanto, no intra a freqüência tendeu a ser menor (12,9\%) (Figura 3).

L. longipalpis e $L$. whitmani foram encontradas sugando sangue humano dentro das casas. Este comportamento foi observado também em L. wellcomei no peridomicílio. Dados mais detalhados desta associação serão apresentados oportunamente.

Tabela 1 - Números de espécimens de flebotomíneos capturados nos diversos ambientes da Ilha de São Luis, MA, Brasil, 1994-1997.

\begin{tabular}{|c|c|c|c|c|}
\hline Espécies de flebótomos & Intradomicílio & Peridomicílio & Mata & Total \\
\hline B. avellari & & & 21 & 21 \\
\hline L. bourrouli & & & 2 & 2 \\
\hline L. claustrei & & & 3 & 3 \\
\hline L. corossoniensis & & & 2 & 2 \\
\hline L. furcata & & & 4 & 4 \\
\hline L. goiana & & & 1 & 1 \\
\hline L. infraspinosa & & & 54 & 54 \\
\hline L. micropyga & & & 5 & 5 \\
\hline L. olmeca & & & 14 & 14 \\
\hline L. oswaldoi & & & 1 & 1 \\
\hline L. rorotaensis & & & 1 & 1 \\
\hline L. sordellii & & & 37 & 37 \\
\hline L. termitophila & & & 1 & 1 \\
\hline L. trispinosa & & & 9 & 9 \\
\hline L. trinidadensis & & & 4 & 4 \\
\hline L. longipennis & & & 4 & 4 \\
\hline L. choti & & 13 & 21 & 21 \\
\hline L. dendrophila & & 1 & 1 & 2 \\
\hline L. shannoni & & 1 & 1 & 2 \\
\hline L. triacantha & & 1 & 2 & 3 \\
\hline L. wellcomei & & 22 & 6 & 28 \\
\hline L. amazonensis & 13 & 9 & 1 & 23 \\
\hline L. antunesi & 27 & 26 & 67 & 120 \\
\hline L. davisi & 2 & 5 & 2 & 9 \\
\hline L. evandroi & 627 & 544 & 157 & 1.328 \\
\hline L. flaviscutellata & 62 & 62 & 285 & 409 \\
\hline L. fluviatilis & 1 & 1 & 1 & 3 \\
\hline L. hirsutus & 3 & 1 & 1 & 5 \\
\hline L. longipalpis & 2.480 & 11.693 & 814 & 14.987 \\
\hline L. migonei & 2 & 4 & 2 & 8 \\
\hline L. umbratilis & 3 & 7 & 7 & 17 \\
\hline L. whitmani & 701 & 2.313 & 2.426 & 5.440 \\
\hline Total de espécimens & 3.921 & 14.703 & 3.957 & 22.581 \\
\hline (\%) & 17,4 & 65,1 & 17,5 & 100,0 \\
\hline
\end{tabular}




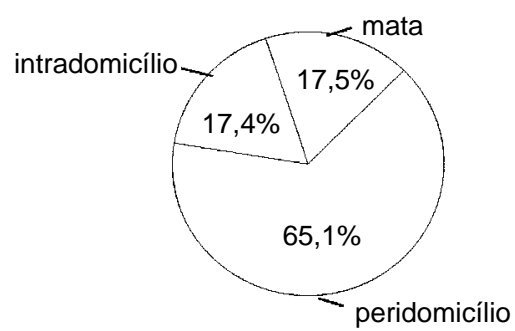

Figura 1 - Números percentuais de espécimens de flebotomíneos capturados nos diversos ambientes na llha de São Luís, MA, de1994 a 1997.

L. evandroi, também apresentou elevada freqüência $(5,9 \%)$, sendo mais abundante no intra (47,2\%) e peridomicílio (40,9\%) (Figura 3), não se detectando diferenças significativas entre estes ambientes $(p>0,05)$.

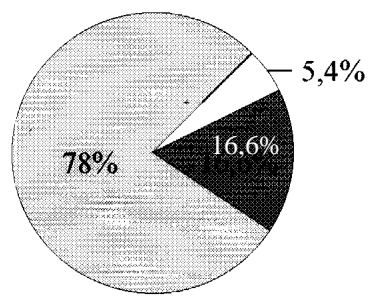

Lutzomyia longipalpis

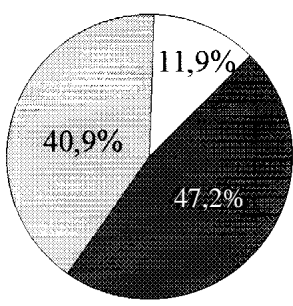

Lutzomyia evandroi

Intradomicílio
$(1,8 \%)$

Lutzomyia flaviscutellata

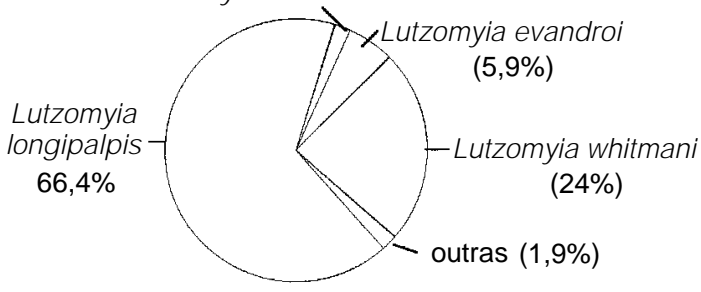

Figura 2 - Números percentuais de espécimens de flebotomíneos capturados na llha de São Luís, MA, de1994 a 1997.

L. flaviscutellata foi significativamente mais freqüente na mata $(p<0,05)$ (Figura 3$)$, onde $69,8 \%$ dos seus exemplares foram capturados, os outros $30,2 \%$ distribuíram-se igualmente nos ambientes peri e intradomiciliar.

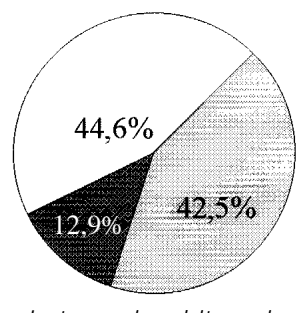

Lutzomyia whitmani

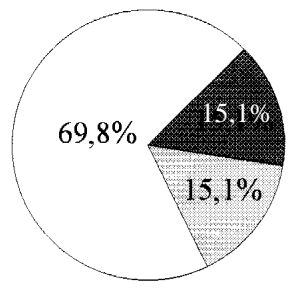

Lutzomyia flaviscutellata

Peridomicílio $\square$ Mata

Figura 3 - Números percentuais de espécimens de flebotomíneos capturados na llha de São Luís-MA, de1994 a 1997.

\section{DISCUSSÃO}

Rebêlo et al12 publicaram a lista das espécies de flebótomos conhecidas no Estado, até 1996, valendo-se de dados da Fundação Nacional de Saúde (FNS), complementados com informações relatadas na literatura por Martins et al10 e Alvim et al2. Nestes trabalhos os autores já haviam mencionados sobre a existência de algumas espécies da Ilha de São Luis.

No entanto, os dados que agora dispõe-se possibilitaram uma nova abordagem sobre a fauna flebotomínica da llha. Os quatro anos de desenvolvimento dos trabalhos de capturas de 
flebótomos associados com a experiência dos técnicos da FNS-MA em suas atividades de rotina pelo interior da llha, leva a acreditar que a fauna local encontra-se razoavelmente conhecida, tanto nas áreas silvestres quanto nos ambientes domiciliares. Trata-se de uma fauna rica e diversificada, com várias espécies restritas ao ambiente silvestre e outras sendo registradas em áreas profundamente alteradas pela ação do homem, incluindo a periferia da zona urbana, como tem-se observado em São Luis.

Entre os flebótomos encontrados na llha de São Luis destacam-se L. longipalpis, L. whitmani, L. wellcomei, L. flaviscutellata e $L$. umbratilis que são reconhecidamente vetores das leishmanioses em suas várias formas. As duas primeiras foram encontradas sugando sangue humano no ambiente domiciliar.

L. longipalpis é a mais freqüente das espécies encontradas no ambiente domiciliar e talvez, a melhor adaptada ao convívio com o homem e os animais domésticos. Ela se encontra distribuída em quase todo a llha, inclusive nas zonas periféricas dos aglomerados urbanos, predominando sobre as demais espécies e fazendo com que a LV assuma um caráter urbano. Sua presença foi registrada, inclusive, nas proximidades da orla litorânea, ao nível do mar e em locais descampados com franca ventilação, fugindo desse modo, das características epidemiológicas apresentadas nas demais localidades do neotrópico2 3.

L. whitmani apresenta grande flexibilidade comportamental, dependendo da região, pode ser silvestre ou peridomiciliar ou ser encontrada nos dois tipos de ambiente. É talvez a espécie mais uniformemente distribuída no Estado12. $\mathrm{Na}$ Ilha de São Luis, tem sido encontrada em todos os ambientes e pode está utilizando o homem, animais domésticos e silvestre como fonte alimentar e transmitindo a eles a $L$. braziliensis e/ou L. shawi, ambas presentes no Maranhão5.

L. wellcomei é também uma espécie epidemiologicamente importante, uma vez que vem sendo incriminada na transmissão da L. braziliensis nas áreas amazônicas ${ }^{13}$. No nosso estudo foi registrada no peridomicílio e no ambiente silvestre. Alguns exemplares foram capturados enquanto sugavam sangue humano e de suíno, no local em que as armadilhas luminosas encontravam-se instaladas.

L. flaviscutellata é considerada estritamente silvestre, com pouca antropofilia, de hábito alimentar essencialmente noturno e ocorrência principalmente em florestas residuais baixas, geralmente úmidas e de difícil acesso para o homem ${ }^{9}$. Na llha de São Luis esta espécie tem sido observada com maior freqüência nas áreas florestais, mas pode ser encontrada nas áreas úmidas de concavidade onde predominam os juçarais (Euterpe oleraceae), conforme observado por nossa equipe. O encontro dela no peridomicílio e dentro das habitações na llha, serve de alerta, pois este flebotomíneo é o vetor da Leishmania amazonensis, o agente responsável pela maioria dos casos de leishmaniose cutânea difusa (LCD) no Estado do Maranhão5.

L. umbratilis tem importante papel como vetora, na região amazônica, da $L$. guyanensis, um dos agentes etiológicos da LTA que até o momento não foi registrado no Maranhão. Mesmo assim, a simples presença deste flebotomíneo em nosso meio é motivo de preocupação.

Entre aquelas espécies que têm sua distribuição restrita aos ambientes de matas da Ilha, destaca-se L. olmeca. É sabido que este flebótomo atua como vetor secundário da L. amazonensis em certas áreas amazônicas e, a exemplo de $L$. flaviscutellata, pode vir a ter algum significado na transmissão de LCD no Maranhão5.

Chama-se a atenção que a metade das espécies estudadas foram encontradas no peridomicílio associando-se, algumas delas, com mamíferos e aves ( $L$. flaviscutellata), aves ( $L$. evandroi), suínos ( $L$. choti e L. triacantha) e roedores ( $L$. antunesi), enquanto outras adentraram nas habitações humanas, mostrando uma certa tendência à antropofilia, o que se reveste de grande importância epidemiológica.

A aproximação de certas espécies de flebótomos com áreas domiciliares pode ser uma conseqüência das alterações em seu ambiente natural, especialmente aquelas produzidas pelo homem. Para se ter uma idéia, nas últimas duas décadas os municípios que compõem a Ilha de São Luis, tiveram um grande impulso econômico e uma explosão no crescimento populacional, em virtude da instalação de vários projetos agropecuários, industriais e habitacionais. Como conseqüência houve uma grande busca pela terra e propiciou um intenso êxodo rural. Este processo resultou em um crescimento desordenado da população nos municípios da llha, provocando sobretudo as invasões.

Este conjunto de fatores foi responsável pela retirada de grande parte da cobertura vegetal da Ilha e, talvez, tenha deslocado os animais 
silvestres de grande porte, hospedeiros sanguíneos dos flebótomos, fazendo com que estes procurassem fontes alternativas de sangue no ambiente periurbano. Em conseqüência disso vários surtos epidêmicos de leishmanioses têm ocorrido desde 1982 até o presente momento, envolvendo a LV e a LTA.

\section{REFERÊNCIAS BIBLIOGRÁFICAS}

1. Alencar JE. Leishmaniose visceral no Brasil. Revista de Medicina da Universidade Federal do Ceará 17/18: 129148, 1977-1978.

2. Alvim MC, Soares OR, Gomes CMC, Ferreira MJS. Flebotomíneos da Ilha de São Luis. I - Composição faunística. I Encontro de Pesquisadores do Maranhão. I Mostra de produção Científica da UFMA. São Luis, p.98, 1990a.

3. Alvim MC, Soares OR, Gomes CMC, Ferreira MJS. Flebotomíneos da llha de São Luis. IV - Espécies antropofílicas. I Encontro de Pesquisadores do Maranhão. I Mostra de produção Científica da UFMA. São Luis, p.98, 1990a.

4. Costa CHN, Pereira HF. Epidemia da leishmaniose visceral no Estado do Piauí, Brasil. Revista de Saúde Pública 24: 361-372, 1990.

5. Costa JML, Saldanha ACR, Mello e Silva AC, Serra-Neto A, Galvão CES, Pedroso e Silva CM, Silva AR. Estado atual da leishmaniose cutânea difusa (LCD) no Maranhão. Revista da Sociedade Brasileira de Medicina Tropical 25: 115-123, 1992.

6. Fernandes MC. Comportamento epidemiológico da leishmaniose tegumentar americana no estado do Maranhão, Brasil de 1980 a 1990. Monografia. Departamento de Patologia da Universidade Federal do Maranhão, 1994.

7. Fundação Nacional de Saúde-Ministério da Saúde. Relatório Anual do Serviço de Epidemiologia. São Luis, 1990.

8. Instituto Brasileiro de Geografia e Estatística. Atlas do Maranhão. Edição em Convênio com o Governo do Estado do Maranhão, Rio de Janeiro, 1984.
9. Lainson R. Our present knowledge of the ecology and control of leishmaniasis in the Amazon Region of Brazil. Revista da Sociedade Brasileira de Medicina Tropical 18:47-56, 1985.

10. Martins AV, Morales-Farias EN. Sobre a distribuição geográfica dos flebotomíneos americanos (Diptera, Psychodidae, Phlebotominae). Revista Brasileira de Biologia 32:361-371, 1972.

11. Projeto Radar Gramétrico da Amazônia. Mapas de geologia e geomorfologia. Vol. 3; Folha SB-23-Teresina e parte da Folha SB-24-Jaguaribe. Vol. 3: Folha SA-23São Luis e parte da Folha 24-Fortaleza. Rio de Janeiro, 1973.

12. Rebêlo JMM, Mendes WA, Costa JML, Cavaleiro N. Lista preliminar das espécies do gênero Lutzomyia França, 1924 (Psychodidae, Phlebotominae) do Estado do Maranhão, Brasil. Caderno de Saúde Pública 12:545549, 1996.

13. Shaw JJ, Lainson R. Ecology and epidemiology: New World. In: The leishmaniasis. Academic Press, London 292-363, 1987.

14. Villas Boas EP, Cantanhede EB, Gonçalves GC, Araújo MV, Segundo WRP. Levantamento de 46 casos de leishmaniose tegumentar americana autóctones da llha de São Luis, MA, atendidos no ambulatório do Departamento de Patologia da Universidade Federal do Maranhão, no período de janeiro de 1995 a abril de 1996. Monografia. São Luis, MA, 1996.

15. Young DG, Duncan MA. Guide to the identification and geographic distribution of Lutzomyia sand flies in Mexico, the West Indies; Central and South America (Diptera: Psychodidae). Gainesville, Florida, 1994. 